\title{
Masking of and by tactile pressure stimuli*
}

\author{
ODED ABRAMSKY $†$ and AMIRAM CARMON† \\ Hadassah Medical Center, Hebrew University, Jerusalem, Israel \\ and \\ ARTHUR L. BENTON+† \\ University of Iowa, Iowa City, Iowa 52240
}

Masking of and by tactile pressure stimuli was investigated in six Ss as a function of stimulus intensity (force) and stimulus onset asynchrony. Increase in the force of the masked stimulus and decrease in the force of the masking stimulus were inversely related to the magnitude of masking, as defined by either a relative or an absolute decrease in sensitivity. The introduction of stimulus onset asynchrony produced both forward and backward masking, the latter being of somewhat larger magnitude. Comparisons are made with results obtained in visual metacontrast masking.

Spatial and temporal parameters in the masking of electrocutaneous stimuli by concomitant application of similar stimuli have been investigated by Uttal (1960), Halliday and Mingay (1960), and Schmid (1961), whose findings indicate significant effects of intensity, relations between time of onset of the stimuli, and locus of stimulation. In contrast, parametric studies of masking in the skin senses with mechanical tactile pressure stimuli have rarely been undertaken, although vibrotactile masking has been the subject of recent studies by Gilson $(1969 \mathrm{a}, \mathrm{b})$. Bird (1964), who investigated the masking of pressure sensation by the use of von Frey filaments, was mainly interested in whether or not masking could be demonstrated in normal Ss and in the effect of changing the locus of the masked and masking stimuli. He found that masking was achieved more readily in the arm than in the face when the masked stimulus was just above threshold. This type of masking with the use of von Frey filaments has been widely employed in clinical neurological studies, since masking in areas with sensory defects is shown more readily than in normal areas (Wortis, Bender, \& Teuber, 1948; Critchley, 1949; Bender, Fink, \& Green, 1951). However, these clinical studies were done without supportive parametric data on the effects of the intensity and temporal relations of the

*Adapted from a thesis submitted by the senior author in partial fulfillment of the requirements for the degree of Doctor of Medicine in the Hebrew University-Hadassah Medical School, Jerusalem. Israel. This study was supported in part by Research Grant NS-00616 and Program-Project Grant NS-03354 from the National Institute of Neurological Diseases and Stroke. Neurosensory Center Publication No. 173.

†Address: Department of Neurology, Hadassah Medical Center, P.O. Box 499, Jerusalem, Israel.

+†Address: Department of Neurology, University Hospitals, Iowa City, Iowa 52240 . stimuli which, in any case, are not well controlled with von Frey hairs.

The present investigation of masking of and by tactile pressure stimuli stemmed from our interest in comparing masking functions in the tactile modality with masking functions in other sensory modalities. The study was made possible by the availability of a recently devised apparatus permitting precisely controlled mechanical stimulation of the skin (Carmon \& Dyson, 1967).

\section{METHOD}

\section{Subjects}

Six male Ss (age range: $22-27$ years) served in the two experiments to be reported.

\section{Apparatus}

An electromechanical stimulator (Carmon \& Dyson, 1967) was used. The instrument, which stimulates the skin by dropping and holding weights on it, permits accurate control of the force duration, rate of application, and the area of the stimuli. The stimulator was equipped with a proximity detector (Distansense D-3, Beta Engineering and Development) for continuous monitoring of the height above the skin surface from which the stimuli are dropped, thus permitting control of the kinetic energy of displacement produced by the impact of the stimulus. The height was kept constant at $1.5 \mathrm{~mm}$ in the two experiments. The order of application of the two stimuli, i.e., masked (Md) and masker (Mr), was controlled by Massey Dickinson behavioral programming modules. Three types of stimulations were used, either each stimulus alone or both concomitantly. The order was automatically randomized by gating a random pulse generator to the driver of a counting ring which selected the stimuli. In the second experiment, a time delay (up to $110 \mathrm{msec}$ ) was used in order to create stimulus onset asynchrony. With no delay, the stimuli onset did not vary more than $5 \mathrm{msec}$, as measured by an oscilloscope.

Duration of stimulation was constant at $720 \mathrm{msec}$. As the stimulus was dropped on the skin, stimulation was induced initially by a displacement impact and then by the displacement produced by the resting weight. The initial impact lasted about $35 \mathrm{msec}$, as measured by recording the initial oscillation of the pulse of the proximity detector on Kodak linagraph paper through a UV recorder at a speed of $500 \mathrm{~mm} / \mathrm{sec}$.

Ss responded to the test stimulus (TS) by pressing a foot switch. Stimulations and responses were recorded on two double electromechanical counters which were appropriately gated to the programming equipment. The first counter counted the stimulations by and the responses to TS when given alone. The second counter recorded the stimulations by and the responses to TS when given concomitantly with the masking stimulus (MS).

\section{Procedure}

$S$ was seated with his left hand resting palm up on a table. The stimulation units with the two stimulators were brought over the ventral surface of the forearm. The masked stimulus was located $6 \mathrm{~cm}$ from the wrist, and the masker stimulus $5 \mathrm{~cm}$ closer to the elbow. These locations were varied randomly from time to time within an area of $1 \mathrm{~cm}^{2}$ in order to avoid habituation and fatigue. The stimulators were brought to $1.5 \mathrm{~mm}$ above the skin by adjustments of the micrometric and macrometric spatial controls of the apparatus. Ss were blindfolded throughout the experiments and were fed continuous white noise through a pair of earphones at a level that masked the mechanical clicks and switch closure sounds produced by the instrument. Vibratory cues were eliminated by having the table on which the hand rested separated from the apparatus. Ss were instructed to respond only to TS under the two possible conditions, when applied alone or with MS. Aberrant responses (to MS alone) were less than $0.1 \%$. Twelve 90-min training sessions preceded the first experiment.

\section{EXPERIMENT 1: EFFECTS OF} CHANGES IN FORCE OF STIMULI

In this experiment, two masked stimuli (20 and $160 \mathrm{mg}$ ) and three maskers $(20,40$, and $80 \mathrm{~g})$ were used in all possible (six) combinations. Twelve blocks of trials, two for each combination with 60 stimulations in each block, were given, making a total number of 720 stimulations. One-third of the stimulations were with TS 


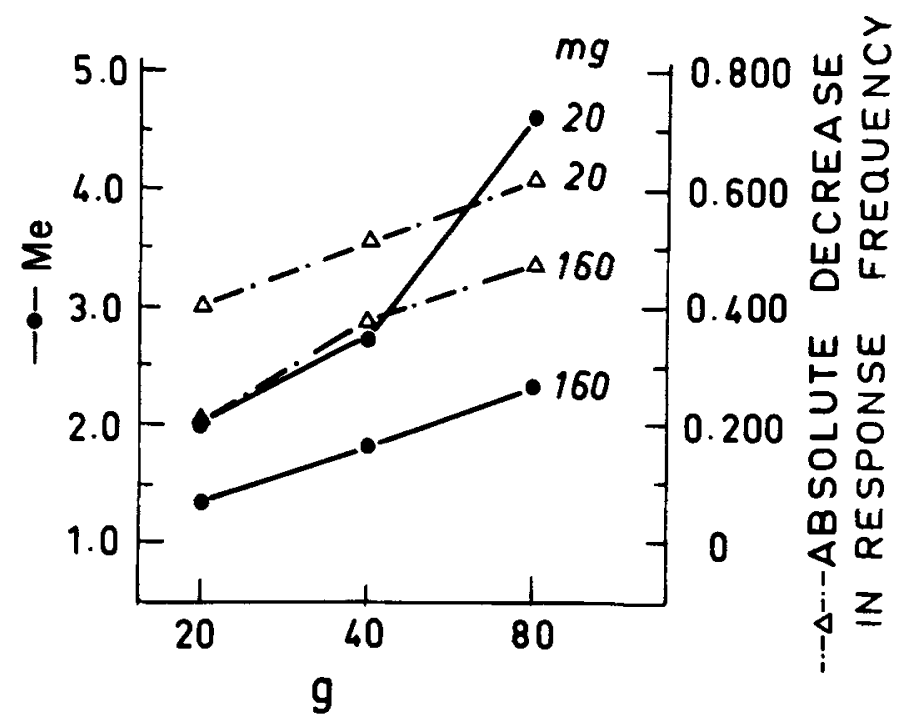

Fig. 1. Masking effect as a function of strength of MS $(20,40,80 \mathrm{~g})$ and TS $(20,160 \mathrm{mg}) ; \mathrm{Me}=$ ratio of number of responses to $\mathrm{TS}$ alone to number of responses to TS when MS is simultaneously applied.

alone, one-third with MS alone, and one-third with both TS and MS. Intertrial intervals ranged from 4 to $10 \mathrm{sec}$ and were varied randomly.

\section{Results}

Sensitivity to each of the two levels of TS was measured by the ratio of the number of responses to the number of stimulations with TS alone. Level of sensitivity to the $20-\mathrm{mg}$ stimulus ranged from .823 to .929 in the six Ss. Level of sensitivity to the $160-\mathrm{mg}$ stimulus ranged from .895 to $\mathbf{9 7 6}$. One hundred percent responding was observed only in two blocks of trials with TS of $160 \mathrm{mg}$ in two Ss. No systematic changes in sensitivity over blocks of trials were observed.

The masking effect (Me) was defined as the ratio of number of responses given to $T S$ alone to the number of responses given to TS when MS was simultaneously applied. Thus Me larger than 1.00 indicated a masking effect, while Me smaller than 1.00 would indicate facilitation of sensitivity to TS by MS.

Masking was observed in all Ss under all TS-MS combinations. A decrease in the intensity of the masked stimulus or an increase in the intensity of the masker stimulus enhanced this effect. The direct relationships between the change in intensity and Me were of unequal magnitude among the six Ss, but all were in the same direction (Fig. 1).

Evaluation of the data was done also by determining the absolute decrease in responses to TS when applied with MS as compared to when TS was applied alone. As shown in
Fig. 1, the results of this comparison were in accord with the results found by using the Me ratio.

\section{EXPERIMENT 2: EFFECT OF STIMULUS ONSET ASYNCHRONY ON MASKING}

In this experiment, constant intensities were used for the masked $(20 \mathrm{mg})$ and for the masker (80 $\mathrm{mg}$ ) stimuli. Stimulus area, location of the stimuli, and the height from which they were dropped were the same as in the first experiment. Fourteen randomized trials were administered, each with 60 stimulations ( 20 TS alone, $20 \mathrm{MS}$ alone, and 20 both). Two trials constituted a block. In three of the seven blocks, the onset of precedes TS; $-=$ MS follows TS.
TS preceded MS by 100,60 , or $30 \mathrm{msec}$. In three other blocks, the onset of MS preceded that of TS by the same time intervals, and in the seventh block, onset was simultaneous. The data were analyzed in the same manner as in the first experiment by calculating $\mathrm{Me}$ and the absolute decrease in response frequency. Both measures yielded the same results (Fig. 2). In addition to the simultaneous masking, which was most prominent, both backward and forward masking were observed, the former effect being slightly larger.

\section{DISCUSSION}

The use of two different measures to evaluate the masking effect, the relative and absolute decrease in responses, yielded similar results. The findings demonstrate the existence of the masking phenomenon in tactile sensitivity to mechanical stimulation. Masking is facilitated by increase in the intensity of the masking stimulus and is maximal when the two stimuli are simultaneous. Increase in the intensity of the masked stimulus or the introduction of stimulus onset asynchrony in either direction decreases the intensity of the masking phenomenon. These findings are in general agreement with studies of masking by electrocutaneous stimuli. Schmid (1961) and Halliday and Mingay (1960) found the same stimulus intensity masking relations. However, while both forward and backward masking were described by Halliday and Mingay, Schmid found for the most part only forward masking in her two Ss.

The extension of the investigation of masking in the skin senses to mechanical stimulation of the skin receptors is important not only because of the demonstration of a

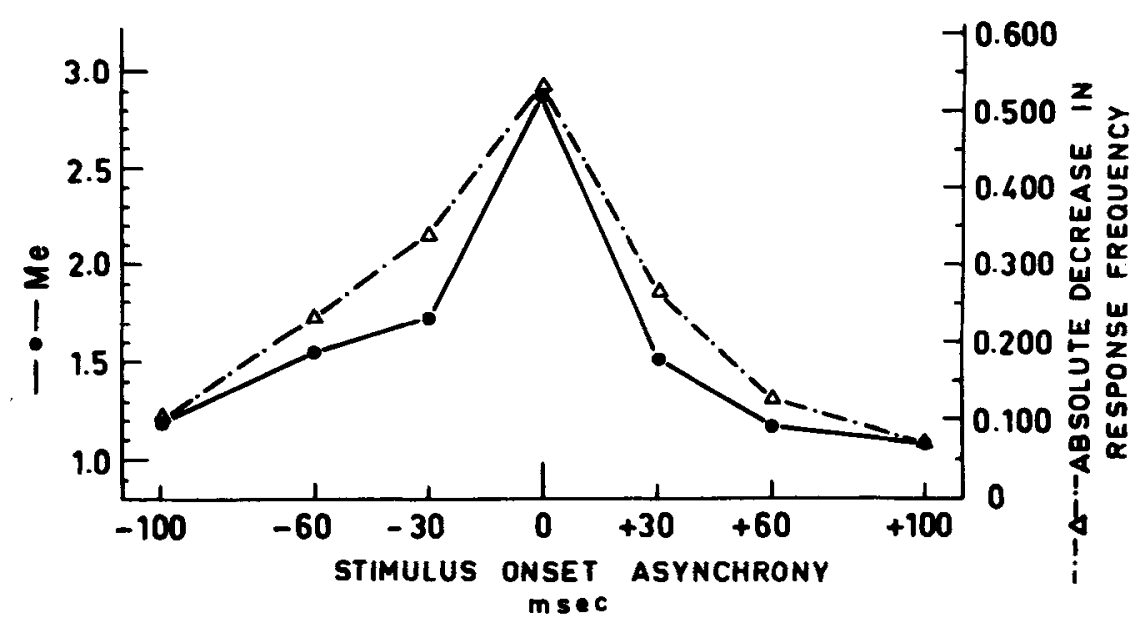

Fig. 2. Masking effect as a function of stimulus onset asynchrony; + =MS 
general sensory phenomenon in still another modality, but also because electrocutaneous stimulation involves some technical problems which are not encountered in mechanical stimulation. The area of stimulation, and thus the number of receptors stimulated, is better defined by a mechanical stimulus which is well circumscribed than by an electrical current which is propagated in an unknown manner. The intensity with which an electrical stimulus activates the skin receptors is also under less control than that of a mechanical stimulus, since the conductance of the skin and the subcutaneous tissues varies and is difficult to measure. Although no direct comparison can be made between the magnitudes of the masking effect achieved by the two methods of stimulation, it is interesting to note that in the studies of Schmid (1961) and Uttal (1960) the current required to achieve masking was at least about twice that of the masked stimulus. These ratios are quite different from those applied in the present experiment. Bird (1964) found that masking of a pressure stimulus could be achieved only if the masked stimulus was just above threshold level and the masker was 9-28 times as strong. Thus his ratios were different from those employed in the studies of Schmid and Uttal and in this investigation.

A problem which is frequently encountered in sensory studies and which might bias the results of a masking experiment is that of the oscillation of tactile sensory thresholds as a function of time. Unless masking, which is in essence an increase in threshold, and the threshold itself are measured at the same time, the outcome of the masking experiment could be interpreted in terms of habituation or fatigue. While Schmid did not make simultaneous determinations of masked and unmasked thresholds, Uttal, by introducing randomly 20 masked and 5 unmasked threshold measurement trials in the same block, was able to overcome this problem. The present experiment followed the same pattern, though we have given the same probabilities of occurrence to both types of trials, thus increasing the reliability of the comparisons. The introduction of still another type of trial, i.e., that with the masking stimulus alone, enabled us to exclude the possibility that the increase in threshold was due to a masking effect which is carried over trials, i.e., habituation. The introduction of prolonged training and the large number of trials in each condition decrease the intra-S variability to a desirable degree. Comparisons between identical trials applied in the beginning and the end of sessions showed no significant difference. However, inter-S variability was quite marked.

Masking, as a function of stimulus intensity and onset asynchrony, has been investigated systematically in the visual and acoustic modalities. A direct comparison with tactile masking is difficult, however, on a methodological basis. Stimuli are not localized in the acoustic receptor system in a way to permit assessment of spatial interaction between stimuli. In vision, comparison can be made only with experiments on masking in metacontrast, i.e., application of a nonoverlapping masked and masker stimulus applied to the same eye. In masking studies of this type, Alpern (1954) and Sperling (1965) found an intensity effect similar to that observed in Experiment 1 of the present study. In their experiment with visual metacontrast, Kolers and Rosner (1960) describe a relationship between stimulus onset asynchrony and masking similar to that found in Experiment 2. These comparisons indicate the existence of analogous perceptual phenomena in the visual and somatosensory systems. Such analogies have also been found between tactile and visual resolution (Carmon \& Benton, in press) and tactile and visual lateral inhibition
(Carmon, 1967), and they reinforce the view that psychophysical findings reflect central processes rather than receptor behavior.

\section{REFERENCES}

ALPERN, M. The effect of luminanes of the contrast inducing flashes on the i, atial range of the metacontrast. Amirican Journal of Optometry. 1954, 31. $363-396$.

BENDER, M. B., FINK, M., \& GREEN, M. Patterns in perception on simultaneous tests of the face and hand. Archives of Neurology \& Psychiatry, 1951, 66, 355-357.

BIRD, J. W. Parameters of double tavtile stimulation. Cortex, 1964, 1, 257-268.

CARMON, A. Stimulus contrast in tactile resolution. Perception \& Psychophysics, $1968,3,241-245$.

CARM ON, A., \& BENTON, A. L Parametric aspects of tactile resolution. Perception \& Psychophysics, 1971, 10, 331-334.

CARMON, A., \& DYSON, J, A. New instrumentation for research on tactile sensitivity and discrimination. Cortex, $1967,3,406-418$.

CRITCHLEY, M. The phenomenon of tactile inattention with special reference to parietal lesions. Brain, 1949, 72, 538-561.

GILSON, R. D. Vibrotactile masking: Effects of multiple maskers. Perception \& Psychoph ysics, 1969a, 5, 181-182.

GILSON, R. D. Vibrotactile masking: Some spatial and temporal aspects. Perception \& Psychophysics, 1969b, 5, 176-180.

HALLIDAY, A. M., \& MINGAY, R. Retroactive raising of a sensory threshold by a contralateral stimulus. Quarterly Journal of Experimetnal Psychology, 1960, 13, 1-11.

KOLERS, P. A., \& ROSNER, B. S. On visual masking (metacontrast): Dichoptic observations. American Journal of Psychology, 1960, 72, 2-21.

SCHMID, E. Temporal aspects of cutaneous interaction with two-point electrical stimulation. Journal of Experimental Psychology, 1961, 61, 400-409.

SPERLING, G. Temporal and spatial visual masking. I: Masking by impulse flashes. Journal of the Optical Society of America, 1965, 55, 541-559.

UTTAL, W. R. Inhibitory interaction of responses to electrical stimuli in the fingers. Journal of Comparative \& Physiological Psychology, 1960, 53. 47-51.

WORTIS, S. B., BENDER, M. B., \& TEUBER, H.-L. The significance of the phenomenon of extinction. Journal of Nervous \& Mental Disease, 1948, 107. 382-387.

(Accepted for publication May 1, 1971.) 